\title{
Exploring the chiral regime with dynamical overlap fermions
}

Hideo Matsufuru* for the JLQCD ${ }^{\dagger}$ and TWQCD Collaborations

High Energy Accelerator Research Organization (KEK), Tsukuba 305-0801, Japan

E-mail: hideo.matsufuru@kek.jp

I report the status of the dynamical overlap fermion project by the JLQCD and TWQCD collaborations. So far, the simulations have been completed with two flavors of overlap sea quarks in a wide range of sea quark mass corresponding to the $p$-regime and the $\varepsilon$-regime on a $16^{3} \times 32$ lattice at $a=0.12 \mathrm{fm}$. More recently, runs with $2+1$ flavors of sea quarks have also started. This talk mainly discusses the physics results on the $N_{f}=2$ lattice after describing the lattice formulation and algorithms.

The XXV International Symposium on Lattice Field Theory

July 30 - August 42007

Regensburg, Germany

${ }^{*}$ Speaker.

$\dagger$ †LQCD Collaboration: S. Aoki, H. Fukaya, S. Hashimoto, K-I. Ishikawa, K. Kanaya, T. Kaneko, H. M., J. Noaki, E. Shintani, M. Okawa, T. Onogi, A. Ukawa, N. Yamada, T. Yoshié.

†TWQD Collaboration: T-W. Chiu, T-H.Hsieh, K. Ogawa. 


\section{Introduction}

Recent development of algorithms and computational resources has enabled extensive studies of dynamical lattice QCD simulations at small quark masses where the chiral symmetry plays an essential role. Such studies are indispensable to investigate the chiral dynamics of QCD and to determine hadronic matrix elements with the precision required by present and future flavor physics. Among lattice fermion actions, the overlap fermion $[1,2]$ has attractive features for these purposes. The overlap fermion operator is represented as

$$
D=m_{0}\left[1+\gamma_{5} \operatorname{sign}\left(H_{W}\left(-m_{0}\right)\right)\right],
$$

where $H_{W}=\gamma_{5} D_{W}$ is the hermitian Wilson-Dirac operator with a large negative mass $-m_{0}$. This operator satisfies the Ginsparg-Wilson relation [3], and thus holds an exact chiral symmetry on the lattice $[4,5]$. The exact chiral symmetry significantly simplifies the structure of operator mixing in calculations of the matrix elements. The overlap fermion corresponds to the infinite $N_{s}$ limit of the domain-wall fermion, which means that one does not have to take care of the residual mass. On the other hand, numerical implementation of the overlap fermion is expensive, because of the evaluation of the sign function of $H_{W}$. Furthermore, the discontinuity of the operator at $\lambda=0$, where $\lambda$ is an eigenvalue of $H_{W}$, makes the molecular-dynamics evolution much involved. Therefore, dynamical simulations of the overlap fermion have become feasible only recently with improved algorithms and large computational power.

We are running a large-scale lattice QCD simulation project with 2 and 2+1 flavors of dynamical overlap fermions. Our physics goals are to explore the chiral regime of QCD with an exact chiral symmetry, and to compute hadronic matrix elements with controlled chiral extrapolation. Present simulations are performed with a spatial lattice size of 16 and $a \simeq 0.12 \mathrm{fm}$. For the gauge action, we adopt the Iwasaki's renormalization group improved action, as well as a topology fixing term which suppresses near-zero modes of $H_{W}[6,7,8]$. Thus our simulation is performed in a fixed topological charge sector. By avoiding the discontinuity of the overlap operator at $\lambda=0$, the numerical cost of HMC is significantly reduced.

This report explains our strategy of simulations and presents several recent results. In the next section, we address why the fixed topology simulation is feasible, and how it can extract physical observables with controlled systematic errors. Section 3 describes the numerical algorithms which are essential for dynamical overlap simulations. In Section 4, our simulation set-up is summarized. Some of recent results at $N_{f}=2$ are shown in Section 5. The last section is devoted to a conclusion and outlook.

\section{Simulations at fixed topology}

\subsection{Topology fixing term}

Dynamical simulations of the overlap fermions are quite nontrivial, because the sign function in Eq. (1.1) has a discontinuity at $\lambda=0$, where $\lambda$ is an eigenvalue of $H_{W}$. Vanishing $\lambda$ may occur during molecular-dynamics steps of the HMC update, at which the topological charge of the system changes its value. To keep the acceptance rate of HMC, one needs to take care of this 
discontinuity so as to conserve the Hamiltonian precisely. One option is the reflection/refraction prescription [9]. This method first determines the time step at which $\lambda$ vanishes, and there the change of the pseudofermion action is computed. Like the light traveling across a surface of water, if the momentum of the mode is larger than the change of the pseudofermion action, it is refracted, while otherwise reflected. This method requires additional inversions of $D(m)$ at $\lambda=0$, and thus the numerical cost quickly increases when the low-mode density becomes large.

We instead employ a topology fixing term, which is implemented with an extra Wilson fermion and a twisted mass ghost as $[6,7,8]$

$$
\begin{gathered}
\operatorname{det}\left(\frac{H_{W}^{2}}{H_{W}^{2}+\mu^{2}}\right)=\int \mathscr{D} \chi^{\dagger} \mathscr{D} \chi \exp \left(-S_{E}\right), \\
S_{E}=\chi^{\dagger}\left[\left(D_{W}+i \gamma_{5} \mu\right)\left(D_{W}^{\dagger} D_{W}\right)^{-1}\left(D_{W}+i \gamma_{5} \mu\right)^{\dagger}\right] \chi .
\end{gathered}
$$

This term is irrelevant in the continuum limit, and considered as a part of the gauge action. The numerator of Eq. (2.1) suppresses near-zero modes of $H_{W}$, while contribution from large frequency modes are compensated by the denominator. Since $\lambda=0$ is prohibited, the topological charge is fixed during the molecular-dynamics update, and hence the reflection/refraction is no longer needed. We set the twisted ghost mass $\mu=0.2$ throughout this work. As was shown in Refs. [8, $10,11]$, this term successfully suppresses the near-zero modes of $H_{W}$.

As an alternative approach, the tunneling HMC has been proposed recently in Ref. [12]. This method also employs the extra Wilson fermion term, and thus near-zero modes of $H_{W}$ are suppressed, but it projects out a few lowest-lying modes during the molecular-dynamics steps so as to enable topology changes. The tunneling HMC does not avoid $\lambda=0$, and the reflection/refraction prescription is necessary. Practical feasibility test is to be performed.

\subsection{Simulations at fixed topology}

Simulations at fixed topology are especially useful in the $\varepsilon$-regime where $1 / m_{\pi} \gg L$ (see Sec. 5.1). In the $\varepsilon$-regime, the topological charge dependence of physical observables is manifest and the fixed topology simulation is useful to determine the low-energy constants appearing in the effective chiral Lagrangian. On the other hand, in the ordinary regime ( $p$-regime), precision calculations are possible only when the following two conditions are satisfied; (1) A physical observable in the $\theta$ vacuum is related to those in the fixed- $Q$ vacua as a systematic expansion in terms of $V^{-1}$, where $V$ is the spacetime volume. (2) The topological susceptibility, $\chi_{t}=\left\langle Q^{2}\right\rangle / V$, is calculable and reproduces the known behavior from ChPT, which ensures that the local fluctuation of topological charge is active so as to produce relevant physics in a finite volume. Here we explain these points along Refs. [13, 14, 15].

What we want to compute is an expectation value in the $\theta$ vacuum, which is related to the "vacua" with fixed values of $Q$ through the Fourier transformation,

$$
Z(\theta)=\sum_{Q} e^{-i \theta Q_{Z}} Z_{Q}, \quad Z_{Q}=\int_{-\pi}^{\pi} \frac{d \theta}{2 \pi} e^{i \theta Q} Z(\theta) .
$$

While the cluster property does hold for the former, the global topology becomes irrelevant in the infinite volume limit and the local fluctuation of topological charge is responsible to the physics. 
In practice, however, the volume is inevitably finite. For a large enough volume, $\chi_{t} V \gg 1$, and for $Q \ll \chi_{t} V$, the saddle point analysis is applicable and it leads to the conclusion that the distribution of $Q$ is Gaussian and physical observables are represented as $\langle O\rangle_{Q}=\langle O\rangle_{\theta}+$ (finite V corrections). As an explicit example, a CP even correlator is represented as

$$
G_{Q}=G(0)+G^{(2)}(0) \frac{1}{\chi_{t} V}\left[1-\frac{Q^{2}}{\chi_{t} V}-\frac{c_{4}}{2 \chi_{t}^{2} V}\right]+G^{(4)}(0) \frac{1}{8 \chi_{t}^{2} V^{2}}+O\left(V^{-3}\right),
$$

where $G^{(n)}(\theta)$ is the $n$-th derivative of $G(\theta)$ with respect to $\theta$, and $c_{4}=-\left(\left\langle Q^{4}\right\rangle-3\left\langle Q^{2}\right\rangle^{2}\right) / V$. To quantify the $O(1 / V)$ effect in Eq. (2.4), $G^{(2)}$ must be known. This is determined with a help of the chiral perturbation theory (ChPT). For example, the $O\left(V^{-1}\right)$ fixed topology effect to the PS meson mass is calculated in Ref. [13] at the tree-level of ChPT. It is also possible to determine $G^{(2)}$ in numerical simulations by comparing the results in different $Q$ sectors.

In the above formula, the topological susceptibility $\chi_{t}$ plays a key role. For a self-contained calculation, $\chi_{t}$ must be computed on the fixed- $Q$ configurations. $\chi_{t}$ is represented as a correlation of the local topological charge $\omega(x)$, which is also subject to Eq. (2.4). From the clustering property in the $\theta$ vacuum,

$$
\lim _{|x| \rightarrow \infty}\langle\omega(x) \omega(0)\rangle_{Q}=\frac{1}{V}\left(\frac{Q^{2}}{V}-\chi_{t}-\frac{c_{4}}{2 \chi_{t} V}\right)+\mathscr{O}\left(V^{-3}\right) .
$$

Using the axial Ward-Takahashi identity,

$$
\lim _{|x| \rightarrow \infty}\left\langle m P^{0}(x) m P^{0}(0)\right\rangle_{Q}=\lim _{|x| \rightarrow \infty}\langle\omega(x) \omega(0)\rangle_{Q}
$$

where $P^{0}(x)$ is the flavor singlet pseudoscalar density. Thus the topological susceptibility can be extracted from the long range behavior of the correlation function carrying the quantum number of $\eta^{\prime}$ meson. If the determined $\chi_{t}$ in a simulation exhibits a reasonable value, it implies that in that system the local fluctuation of topological charge is active enough, and the system size is sufficiently large. Extraction of $\chi_{t}$ in our $N_{f}=2$ simulation will be described in Sec. 5.2. Such a method is useful not only in the fixed topology simulations but also in standard HMC updates, because the changes of topological charge with a continuous variation of link variables become increasingly rare as approaching the continuum limit.

These arguments indicate that the fixed topology simulations can provide a framework to determine the physical observables in the $\theta$ vacuum in a self-contained manner with the finite size effects under control.

\section{Algorithm}

\subsection{Overlap operator}

The overlap operator with a quark mass $m$ is written as

$$
D(m)=\left(m_{0}+\frac{m}{2}\right)+\left(m_{0}-\frac{m}{2}\right) \gamma_{5} \operatorname{sign}\left[H_{W}\left(-m_{0}\right)\right] .
$$

$m_{0}$ is set to 1.6 throughout this work. The sign function means that an eigenmode $\left(\lambda, v_{\lambda}\right)$ of $H_{W}$ is transformed to $\left(\operatorname{sign}(\lambda), v_{\lambda}\right)$. Since the calculation of all the eigenmodes is impractical, some kind 
of approximation of the sign function is required. We employ the Zolotarev rational approximation $[16,17]$,

$$
\frac{1}{\sqrt{H_{W}^{2}}}=\frac{d_{0}}{\lambda_{\min }}\left(h_{W}^{2}+c_{2 n}\right) \sum_{l=1}^{N} \frac{b_{l}}{h_{W}^{2}+c_{2 l-1}},
$$

where $h_{W}=H_{W} / \lambda_{\min }$ with $\lambda_{\min }$ the eigenvalue having the smallest absolute value. $d_{0}, c_{l}, b_{l}$ are easily calculable parameters. Although $\left(h_{W}^{2}+c_{2 l-1}\right)^{-1}$ must be calculated $N$ times, these terms can be obtained simultaneously by the multi-shift CG method $[18,19]$. Thus, the numerical cost mildly depends on $N$. This formula is valid in the region $|\lambda| \in\left[\lambda_{\min }, \lambda_{\max }\right]$. Since the error of the formula scales as $\exp \left(-\lambda_{\text {min }} N\right)$, the smaller $\lambda_{\text {min }}$ requires the larger $N$ to keep the precision unchanged. Instead, if one calculates low-lying eigenvalues of $H_{W}$ of $|\lambda|<\lambda_{\text {thrs }}$, one can determine the sign function of these modes explicitly and project them out from $H_{W}$. Then $\lambda_{\text {thrs }}$ replaces $\lambda_{\min }$ in the above formula, leading to

$$
\operatorname{sign}\left(H_{W}\right)=\sum_{j=1}^{N_{e v}} \operatorname{sign}\left(\lambda_{j}\right) v_{j} \otimes v_{j}^{\dagger}+\operatorname{sign}\left(H_{W}\right) P_{H},
$$

where $P_{H}=1-\sum_{j=1}^{N_{e v}} v_{j} \otimes v_{j}^{\dagger}$, and $N_{e v}$ the number of modes with $\left|\lambda_{j}\right|<\lambda_{\text {thrs }}$. The approximation formula is applied to the second term of this equation. The numerical cost depends on the density of low modes, since the determination of the low modes requires non-negligible computational time, and also projecting out the low modes from the sign function requires additional operations. Also in this sense, employing the topology fixing term improves the simulations of overlap fermions. In this work, we adopt $\lambda_{\text {thrs }}=0.045$ and $N=10$, which lead to an accuracy of $\left|\operatorname{sign}^{2} H_{W}-1\right| \simeq 10^{-(7-8)}$.

\subsection{Solver algorithms}

Since the overlap operator must be inverted at each step of the molecular-dynamics evolution, the improvement of the solver algorithm may significantly reduce the simulation cost. We have tested two algorithms; the nested CG (or 4DCG) method [20] and the 5-dimensional CG (5DCG) method [21, 22]. The nested CG method is a straightforward implementation of the overlap solver. It contains two nested CG iterations: an outer loop for operating $D(m)$ and an inner loop for the calculation of $\left(H_{W}^{2}+c_{2 l}\right)^{-1}$. The numerical cost can be reduced by applying the relaxation technique, which relaxes the convergence criterion of the inner loop as the outer loop iteration proceeds. Instead of the CG method, one can apply other Krylov subspace algorithms such as GMRES and SUMR. Since in HMC only the inversion of $D^{\dagger} D$ appears and the CG method almost achieves the best performance, we compare the CG method with the 5D algorithm in the following.

The 5-dimensional CG solver is based on the Schur decomposition [21, 22]. Let us consider a 5-dimensional block matrix (the $N=2$ case is displayed as an example),

$$
M_{5}=\left(\begin{array}{cccc|c}
H_{W}-\sqrt{q_{2}} & & & 0 \\
-\sqrt{q_{2}}-H_{W} & & & \sqrt{p_{2}} \\
& H_{W} & -\sqrt{q_{1}} & 0 \\
& -\sqrt{q_{1}}-H_{W} & \sqrt{p_{1}} \\
\hline 0 & \sqrt{p_{2}} & 0 & \sqrt{p_{1}} & R \gamma_{5}+p_{0} H
\end{array}\right)=\left(\begin{array}{c|c}
A & B \\
\hline C & D
\end{array}\right) .
$$



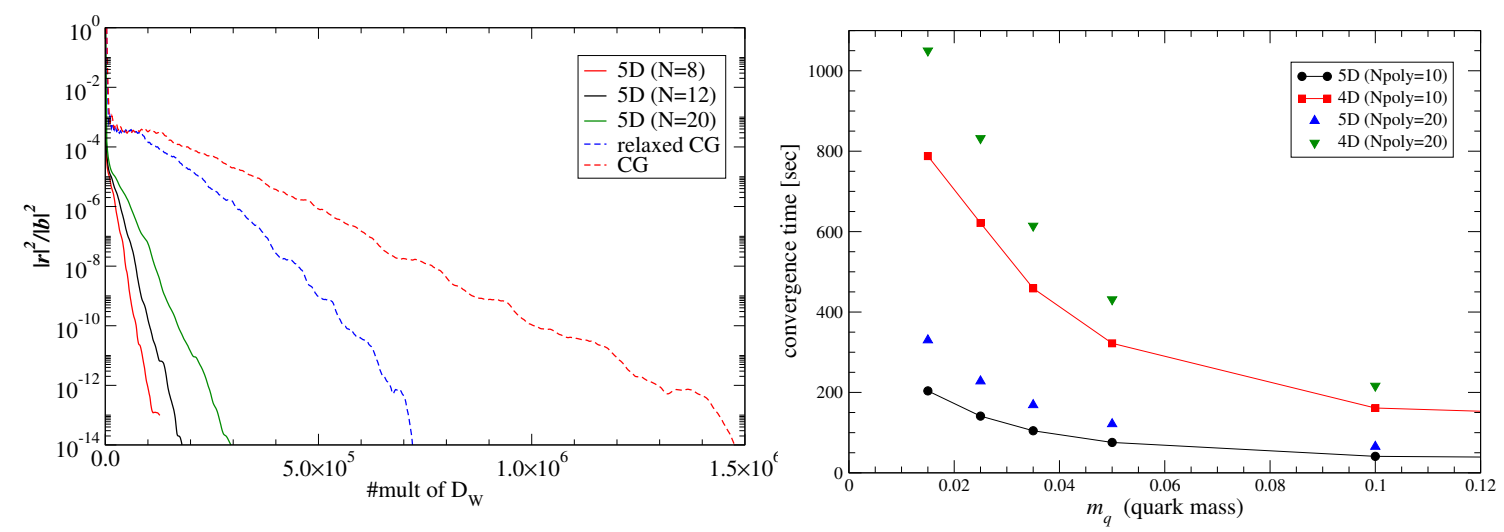

Figure 1: Comparisons of the overlap solvers. The left panel shows the residual of the CG solver without the low-mode projection. The right panel shows the required time for the convergence in the case with the projection of 8 low modes of $H_{W}$.

Since $M_{5}$ can be decomposed as

$$
M_{5}=\left(\begin{array}{cc}
1 & 0 \\
C A^{-1} & 1
\end{array}\right)\left(\begin{array}{ll}
A & 0 \\
0 & S
\end{array}\right)\left(\begin{array}{ll}
1 & A^{-1} B \\
0 & 1
\end{array}\right),
$$

where $S=D-C A^{-1} B$ is called the Schur complement. One can solve a $4 \mathrm{D}$ linear equation $S \psi_{4}=$ $\chi_{4}$ by solving a $5 \mathrm{D}$ equation

$$
M_{5}\left(\begin{array}{c}
\phi \\
\psi_{4}
\end{array}\right)=\left(\begin{array}{c}
0 \\
\chi_{4}
\end{array}\right) .
$$

Setting the parameters $R, p_{0}, p_{i}$ and $q_{i}(i=1, \ldots, N)$ in Eq. (3.4) appropriately, the 5D solver can be used to invert the overlap operator approximated by Eq. (3.2). The 5D solver is accelerated by applying the even-odd preconditioning. Then one needs to solve a reduced linear equation, $\left(1-M_{e e}^{-1} M_{e o} M_{o o}^{-1} M_{o e}\right) \psi_{e}=\chi_{e}^{\prime}$, where even/odd blocks of $M_{5}$ is denoted by $M_{e e}, M_{e o}$, etc. The inversions $M_{e e}^{-1}$ and $M_{o o}^{-1}$ are easily calculated by forward/backward substitutions. The projection of low-modes is not straightforward for the even-odd preconditioned 5D solver, since the operation of $M_{e e}^{-1}$ (or $M_{o o}^{-1}$ ) becomes much involved. Nevertheless it can be implemented cheaply, because the subspace of the matrix is spanned by $x_{e}, \gamma_{5} x_{e}, v_{j e}, \gamma_{5} v_{j e}\left(j=1, \ldots, N_{e v}\right)$ [23].

Figure 1 compares the 4D and 5D solvers. The left panel shows the behavior of the residual against the numbers of the Wilson-Dirac operator multiplications, in the case of no low-mode projection [28]. The relaxation accelerates the nested CG by a factor of two, while the 5D solver exhibits much faster convergence for practical values of $N$. The required time to solve the linear equation is compared in the right panel for the case that the number of projected low modes is 8 . This shows that the 5D solver is 3-4 times faster than the 4D solver in the whole region of quark mass used in this work. Therefore we mainly use the 5D solver in HMC. For the computation of the quark propagator, we adopt the nested 4D solver because of an advantage to obtain the propagators with several valence quark masses simultaneously with the multi-shift CG algorithm.

Here let us compare the cost of simulations with the domain-wall fermion. Let us consider the cost to solve a linear equation $D x=b$. At $m \simeq m_{s} / 2, a^{-1} \simeq 1.7 \mathrm{GeV}$, and on $16^{3} \times 32$ lattices, the 
domain-wall solver requires $O(800)$ iterations for a precision $|r| /|b|<10^{-10}$ according to Ref. [24], while the overlap solver requires $O(1200)$ iterations. To convert to the numbers of the WilsonDirac operator multiplications, $2 N_{s}$ and $2 N+1$ are multiplied for the domain-wall and the overlap fermions, respectively. Then the difference amounts to a factor of about 2.5. In HMC, the overlap solver must be called twice, while the domain-wall solver is applied to $D^{\dagger} D$. This leads to another factor of 2 difference leading to the total difference of $O(5)$.

\subsection{Hybrid Monte Carlo algorithm}

Implementation of the HMC algorithm for the overlap operator with the approximation formula (3.2) is straightforward except for the discontinuity at $\lambda=0$. Since we employ the topology fixing term, $\lambda=0$ does not appear and no reflection/refraction prescription is required. In order to improve the performance of HMC, we adopt the mass preconditioning [25] together with the multi-time step procedure [26]. Introducing a preconditioning term with a heavier quark mass $m^{\prime}$ than the dynamical quark mass $m$, the fermion action becomes $S_{F}=S_{P F 1}+S_{P F 2}$,

$$
S_{P F 1}=\phi_{1}^{\dagger}\left[D\left(m^{\prime}\right)^{\dagger} D\left(m^{\prime}\right)\right]^{-1} \phi_{1}, \quad S_{P F 2}=\phi_{2}^{\dagger}\left\{D\left(m^{\prime}\right)\left[D(m)^{\dagger} D(m)\right]^{-1} D\left(m^{\prime}\right)^{\dagger}\right\} \phi_{2} .
$$

The forces from the preconditioner (PF1), the preconditioned dynamical quark (PF2), the gauge field (G), and the extra Wilson fermion/ghost field (E) have a hierarchical structure,

$$
F_{G} \sim F_{E} \gg F_{P F 1} \gg F_{P F 2}
$$

Thus we set

$$
\Delta \tau_{(P F 2)} \gg \Delta \tau_{(P F 1)} \gg \Delta \tau_{(G)}=\Delta \tau_{(E)}
$$

$\Delta \tau_{(E)}$ is set to be the same as the gauge part also to ensure the disappearance of near-zero modes of $H_{W}$. The cost to calculate $F_{E}$ is negligible compared to the overlap fermions. In the case of $N_{f}=2+1$, the time steps of the two-flavor and one-flavor parts are set equal. In this work, $\Delta \tau_{(P F 2)} / \Delta \tau_{(P F 1)}$ and $\Delta \tau_{(P F 1)} / \Delta \tau_{(G)}$ are set to 4-6.

In the $N_{f}=2$ simulation, we employ the noisy Metropolis test [27] together with a less precise 5D solver without the projection of low-modes of $H_{W}$ [10,28]. During the molecular dynamics, the calculation of the low-lying modes of $H_{W}$ is skipped. We fix the value of $\lambda_{\text {thrs }}$, the lower-bound of the region where the approximation formula (3.2) is valid, though the modes with $|\lambda|<\lambda_{\text {thrs }}$ may appear. The error with this setting is corrected by the noisy Metropolis test performed at the end of each MD evolution in addition to the usual Metropolis test. This algorithm is twice faster than the case with the 4D solver with the projection of low-modes of $H_{W}$, which was used in an early stage of the simulation.

The $N_{f}=2+1$ simulation has been started recently [23]. The one-flavor part is implemented with one of the chirality sectors $[29,30]$ making use of the fact that $H(m)^{2}$ commutes with $\gamma_{5}$, thus

$$
H^{2}=P_{+} H^{2} P_{+}+P_{-} H^{2} P_{-} \equiv Q_{+}+Q_{-}, \quad \operatorname{det} H^{2}=\operatorname{det} Q_{+} \cdot \operatorname{det} Q_{-} .
$$

Except for the trivial contribution from the zero-modes, the determinant of one chirality sector corresponds to the contribution of one flavor. Thus, the pseudofermion action $S_{F}=\phi_{\sigma}^{\dagger} Q_{\sigma}^{-1} \phi_{\sigma}$, where $\sigma$ can be either + or - , represents the one-flavor of dynamical fermion. The same acceleration 

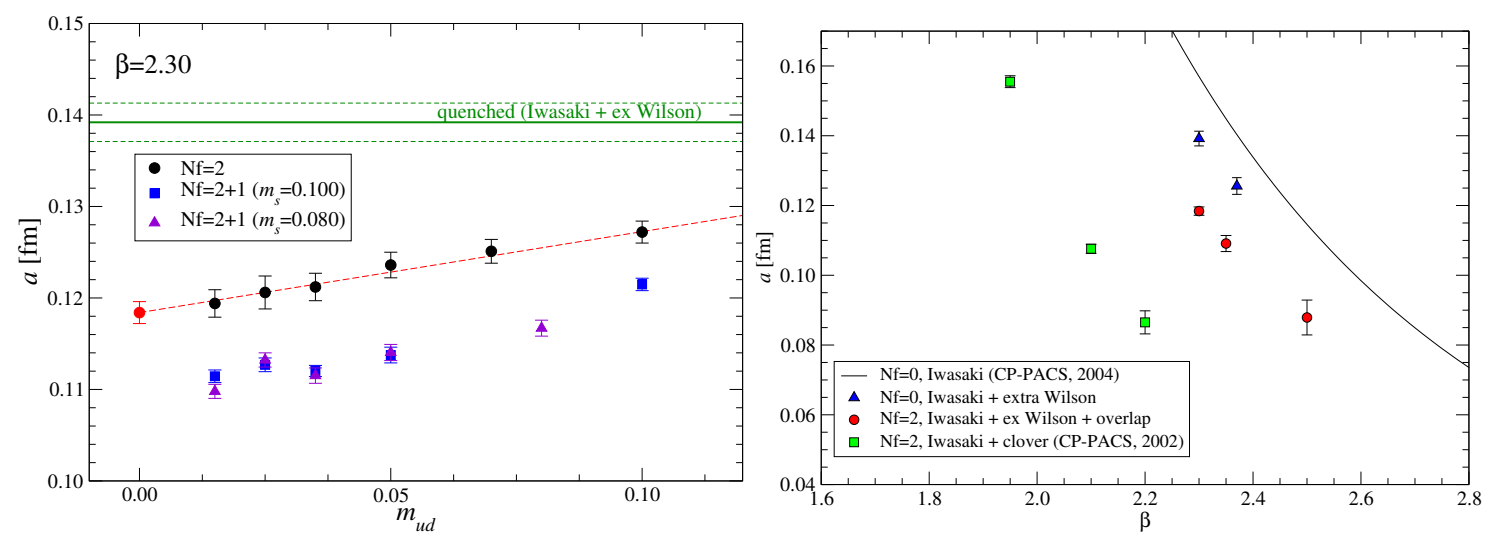

Figure 2: The left panel shows the lattice scale $a\left(r_{0}\right)$ set by $r_{0}=0.49 \mathrm{fm}$. The right panel shows the $\beta$ dependence of $a\left(r_{0}\right)$ for $N_{f}=2$ and 0 , together with the result of the clover fermion.

techniques as $N_{f}=2$ are applicable to the one-flavor part. For the $N_{f}=2+1$ simulation, we adopt the 5D solver with the projection of low-modes of $H_{W}$, which no longer requires the noisy Metropolis test [23].

\section{Simulation}

Numerical simulations are performed mainly on IBM Blue Gene/L system at KEK. At present the sustained performance on one rack of Blue Gene/L (1024 nodes, 5.7 TFlops of peak performance) is about $30 \%$ for the Wilson operator [31], and 10-15\% for the overlap HMC.

The $N_{f}=2$ simulations are performed on $16^{3} \times 32$ lattices at $\beta=2.3$. We use 6 quark masses, $0.015,0.025,0.035,0.050,0.070$, and 0.100 , roughly corresponding to $m_{s}^{\text {phys }} / 6-m_{s}^{\text {phys }} .10,000$ trajectories of a length 0.5 are generated at each sea quark mass in the $Q=0$ sector. For $m_{u d}=0.050$ $\left(\simeq m_{s}^{p h y s} / 2\right)$, configurations in $Q=-2$ and -4 sectors are also generated. In addition, we perform a simulation in the $\varepsilon$-regime at $\beta=2.35$ with a quite small quark mass, $m_{u d} \simeq 3 \mathrm{MeV}$. To generate one trajectory, 11-26 minutes are needed on one rack of Blue Gene/L. The acceptance rate is kept to $80-90 \%$ at each quark mass. The locality of the overlap operator was examined in Ref. [32].

The $N_{f}=2+1$ simulations have been started recently on $16^{3} \times 48$ lattices with almost the same parameters as $N_{f}=2$, while each trajectory has a length 1.0 [23]. We use 5 values of $m_{u d}$ covering the same quark mass region as the $N_{f}=2$ case for each of 2 strange quark masses, $m_{s}=0.080$ and 0.100 , around the physical strange quark mass $m_{s}^{\text {phys }}$. Present performance is around 2 hours for one trajectory on one rack of Blue Gene/L.

The lattice scale is set by the hadronic radius $r_{0}$ which is defined through

$$
\left.r^{2} \frac{V(r)}{\partial r}\right|_{r=r_{0}}=1.65
$$

by setting the physical value $r_{0}=0.49 \mathrm{fm}$. The static quark potential $V(r)$ is calculated with the standard procedure. Figure 2 shows the result of the lattice spacing. The left panel displays the result for $N_{f}=2$ extrapolated to the chiral limit, as well as a preliminary result for $N_{f}=$ $2+1$. A linear extrapolation of the $N_{f}=2$ data gives $a\left(m_{u d}=0\right)=0.1184(12)_{\text {stat }}(11)_{\text {syst }} \mathrm{fm}$. 
The right panel shows the $\beta$-dependence of $a$ in the chiral limit for $N_{f}=0$ and 2 together with the clover fermion case. The shift of $\beta(a)$ with respect to the number of flavors is milder for the overlap fermion than for the Wilson-type fermions. This behavior is consistent with the perturbative calculation.

\section{Results}

On the $N_{f}=2$ lattices, we finished the generation of gauge configurations and are currently calculating various physical observables. The following calculations are in progress.

- $\varepsilon$-regime $[33,34,35,36]$

- Topological Susceptibility $[37,38]$

- Pion mass and decay constant [39]

- Pion form factor [40]

- $B$ meson bag parameter [41]

- $\pi^{+}-\pi^{0}$ mass difference [42]

- Pion scattering length [43]

In the following, we briefly describe the first three subjects, which are the first testing ground of the viability of our simulations.

\section{$5.1 \varepsilon$-regime}

The chiral condensate is related to the spectral density of the Dirac operator through the BanksCasher relation [44],

$$
\Sigma \equiv-\langle\bar{q} q\rangle=\lim _{m \rightarrow \infty} \lim _{V \rightarrow \infty} \frac{\pi \rho(0)}{V},
$$

where $\rho(\lambda)=\sum_{k}\left\langle\delta\left(\lambda-\lambda_{k}\right)\right\rangle$ is the spectral density of the Dirac operator. The accumulation of low modes generate the spontaneous chiral symmetry breaking. Two limits, $m \rightarrow \infty$ and $V \rightarrow \infty$, in the above equation are not commutable. However, it is also convenient to consider the opposite order of the limit. The $\varepsilon$-regime is defined through the condition

$$
1 / \Lambda_{Q C D} \ll L \ll 1 / m_{\pi}
$$

which implies $m \ll 1 / \Sigma V$. In the $\varepsilon$-regime, the low energy effective theory is applied with the same parameters as the infinite $V$ case. Because of the finiteness of the volume, the topological charge dependence of observables becomes manifest, and hence it is convenient to determine the parameters of the low energy effective Lagrangian in the fixed topology simulations. Another advantage of the $\varepsilon$-regime simulations is that the chiral random matrix theory (RMT) is expected to describe the behavior of the low-lying modes.

The results in the $\varepsilon$-regime for $N_{f}=2$ are displayed in Figure $3[34,35]$. We find good agreement with the RMT prediction for the level distribution. The lowest level distribution gives a value of the chiral condensate which is consistent with other determinations. Another agreement with the RMT prediction is found for the so-called topology-flavor duality, as shown in the right panel of Figure 3. With the nonperturbative renormalization, we obtaine

$$
\Sigma^{\overline{M S}}(2 \mathrm{GeV})=(251 \pm 7 \text { (stat) } \pm 11 \text { (syst) } \mathrm{MeV})^{3} .
$$

The main source of the systematic error is $O\left(\varepsilon^{2}\right)$, which can be corrected using meson correlators [36]. 

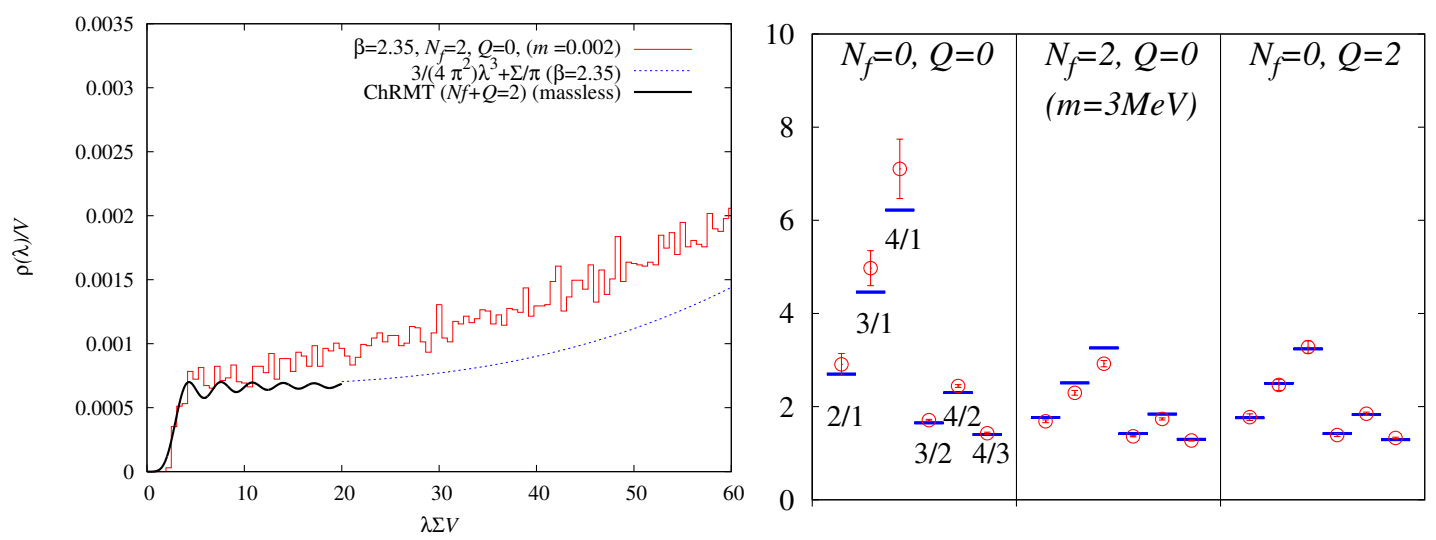

Figure 3: The result in the $\varepsilon$-regime at $N_{f}=2$. The left panel shows the spectral density of the overlap-Dirac operator. The right panel compares the level spacings in the cases of different $Q$ and $N_{f}$.

\subsection{Topological susceptibility}

Now let us go back to the $p$-regime. We first need to show that the topological susceptibility is successfully determined in the fixed- $Q$ "vacua" from the correlation function (Sec. 2.2). The topological susceptibility $\chi_{t}$ is extracted from the correlation function

$$
C_{\eta^{\prime}}(t)=\frac{1}{L^{3}} \sum_{\vec{x}}\left\langle m_{q} P^{0}(x) m_{q} P^{0}(0)\right\rangle_{Q},
$$

where $P^{0}(x)$ is the flavor singlet pseudoscalar density. For the overlap fermions,

$$
P^{0}(x)=\frac{1}{N_{f}} \sum_{f=1}^{N_{f}} \bar{\psi}^{f}(x) \gamma_{5}\left[1-\frac{a D\left(m_{q}=0\right)}{2 m_{0}}\right] \psi^{f}(x) .
$$

At large $t$,

$$
C_{\eta^{\prime}}(t)=\frac{1}{V}\left(\frac{Q^{2}}{V}-\chi_{t}-\frac{c_{4}}{2 \chi_{t} V}\right)+\mathscr{O}\left(V^{-3}\right)+\mathscr{O}\left(e^{-m_{\eta^{\prime}} t}\right),
$$

hence the value of $\chi_{t}$ is extracted from the plateau of $C_{\eta^{\prime}}(t)$.

The measurement of $\chi_{t}$ is performed on $N_{f}=2$ and $Q=0$ lattices at every 20 trajectories, thus with statistics of 500. Prior to measuring the correlator, we calculate 50 pairs of low-lying eigenmodes of the overlap operator $D(0)$ by the implicitly restarted Lanczos algorithm. Since these low-modes can be explicitly inverted, the solver algorithm is applied to the operator projected out these modes, whose condition number is reduced from the original one. This low-mode preconditioning accelerates the calculation of the quark propagators by a factor of 8 . These low-modes are also used in the low-mode averaging, which is an average of correlators over all the spacetime source points for the low-mode contributions [45, 46]. For the disconnected part of the correlator (5.4), the quark propagator is approximated by 50 pairs of the low-eigenmodes, by observing these modes dominate the correlator.

The result is shown in Figure 4. The left panel displays the correlator $-C_{\eta^{\prime}}(t)$ at $m_{q}=0.025$. The data are fitted to a function $A+B\left(e^{-M t}+e^{-M(T-t)}\right)$. Assuming $\left|c_{4}\right| \ll 2 \chi_{t} V$, we obtain $a^{4} \chi_{t}=$ $3.40(27) \times 10^{-5}$ at $m_{q}=0.025$. The right panel shows the extracted $\chi_{t}$ as a function of sea quark 

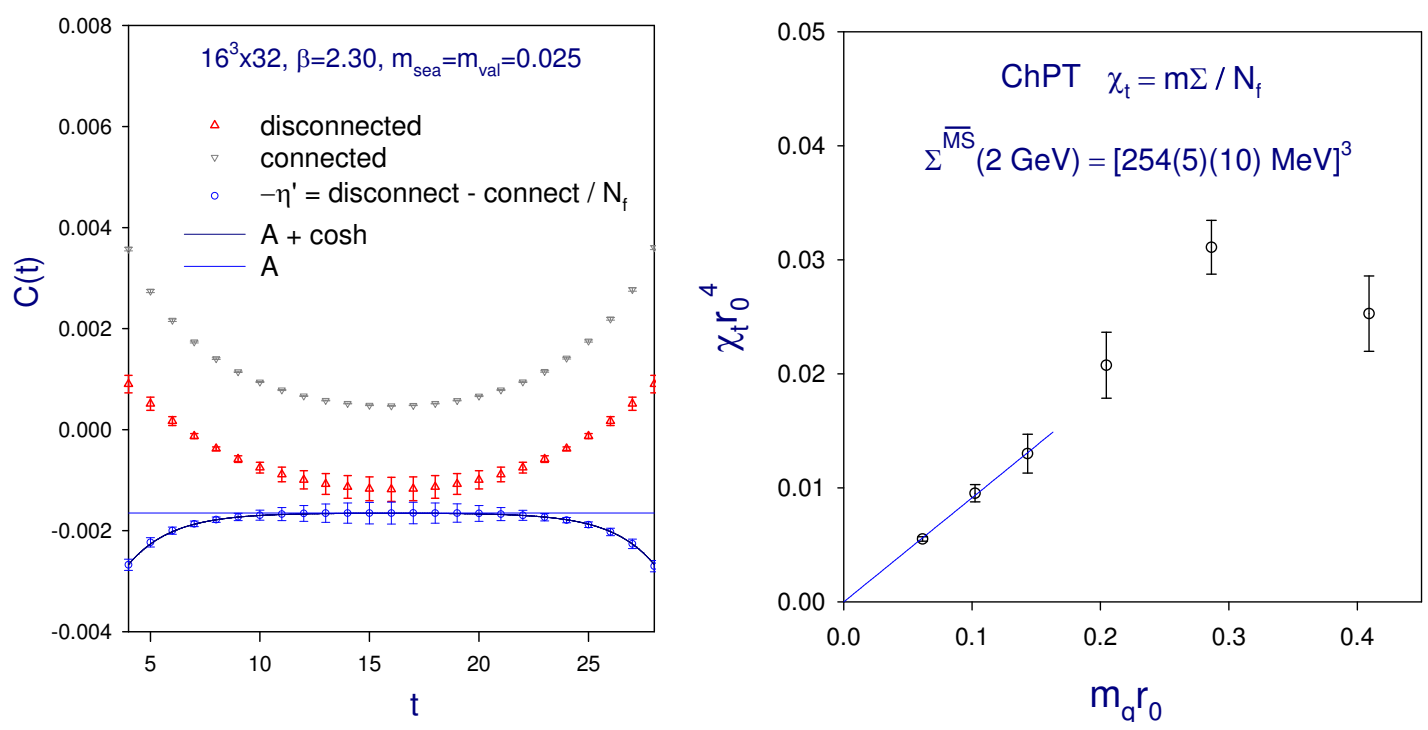

Figure 4: The result of the topological susceptibility for the $N_{f}=2$ simulation. The left panel shows the extraction of the topological susceptibility $\chi_{t}$ from the $\eta^{\prime}$ correlator at $m_{q}=0.025$. The right panel displays $\chi_{t} r_{0}^{4}$ versus the sea quark mass $m_{q} r_{0}$.

mass in units of $r_{0}$. For the smallest three quark masses, the data are well fitted to a linear function whose intercept is consistent with zero. This behavior is consistent with the prediction of the chiral perturbation theory [47],

$$
\chi_{t}=\frac{m_{q} \Sigma}{N_{f}}+O\left(m_{q}^{2}\right)
$$

From the slope of the fit result, a value of the chiral condensate is obtained as $r_{0}^{3} \Sigma=0.182(6)$. In order to convert it to a physical value, we use the renormalization factor $Z_{m}^{\overline{\mathrm{MS}}}(2 \mathrm{GeV})=0.742(12)$, which is obtained nonperturbatively through the RI/MOM scheme on the lattice [48, 39]. This leads to a value

$$
\Sigma^{\overline{M S}}(2 \mathrm{GeV})=(254 \pm 5 \text { (stat) } \pm 10 \text { (syst) } \mathrm{MeV})^{3},
$$

which is consistent with the value in Eq. (5.3) independently obtained in the $\varepsilon$-regime. The statistical error includes those of $a^{-1}$ and $Z_{m}^{\overline{\mathrm{MS}}}$, and the systematic error is of the higher order effects such as the $c_{4}$ term.

We also measure $\chi_{t}$ on the $N_{f}=2$ lattices with $Q=-2$ and -4 at $m_{q}=0.050$ with statistics of 250 configurations. The extracted values of $\chi_{t}$ are consistent with the value at $Q=0$.

These results indicate that the local fluctuation of the topological charge is active enough to produce relevant chiral dynamics on the fixed- $Q$ vacua. The successful extraction of $\chi_{t}$ enables us to quantify the finite size effects for other observables represented in Eq. (2.4).

\subsection{Pion mass and decay constant}

Among hadronic observables, the pion mass and decay constant are the primary quantities to be tested with the chiral perturbation theory. Here we briefly present our results of the $N_{f}=2$ simulation for $m_{\pi}$ and $f_{\pi}[39]$. 

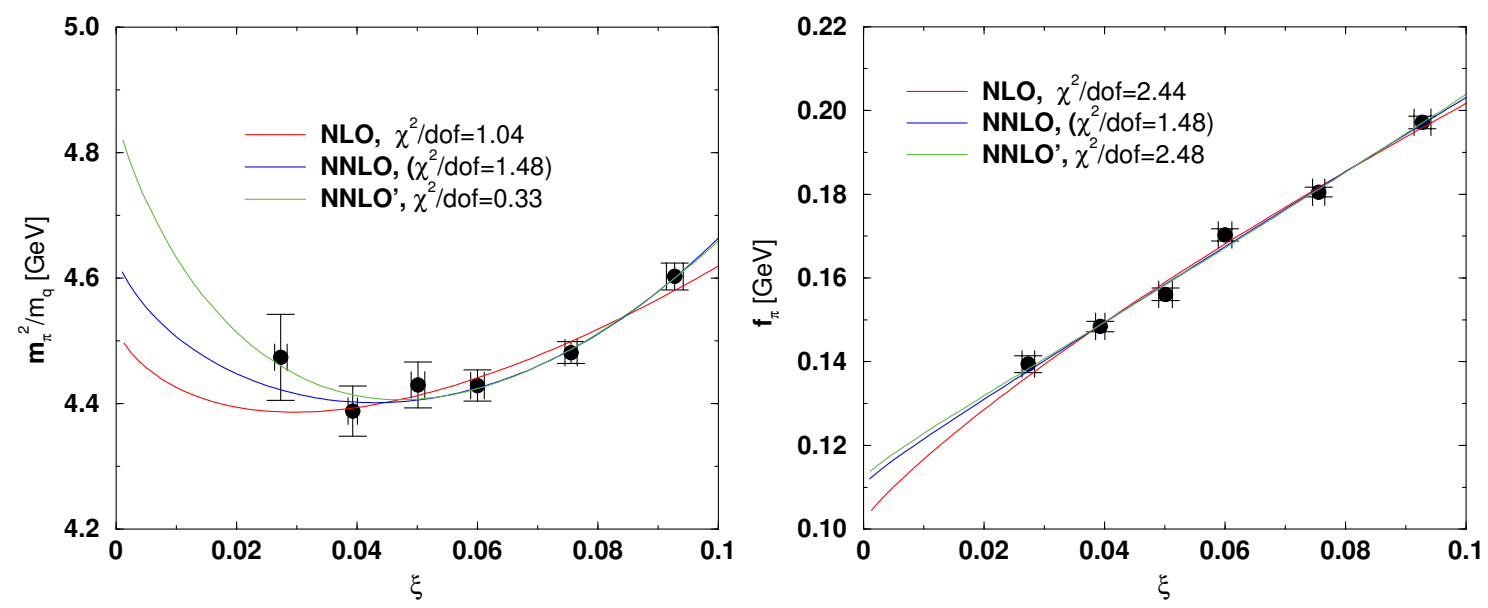

Figure 5: The results of chiral extrapolation for $m_{\pi}^{2} / m_{q}$ (left panel) and $f_{\pi}$ (right) in physical units as functions of $\xi$. Three types of fits, NLO, NNLO, and NNLO' are performed using all the available data points.

The meson correlators are computed at every 20 trajectories. The low-mode preconditioning and the low-mode averaging described in the previous subsection are applied to the meson correlators. The pion mass and decay constant are extracted from the pseudoscalar meson correlators with point and smeared sources by a simultaneous fit. $f_{\pi}$ is obtained through the axial Ward-Takahashi identity,

$$
f_{\pi}=2 m_{q}\langle 0|P(0)| \pi\rangle / m_{\pi}^{2}
$$

without further renormalization. Note that we are using the $f_{\pi}=130 \mathrm{MeV}$ normalization. The quark mass is renormalized with the renormalization factor $Z_{p}^{\overline{\mathrm{MS}}}(2 \mathrm{GeV})=0.742(12)$, which is obtained nonperturbatively through the RI/MOM scheme on the lattice [48, 39].

The pion mass and decay constant receive two kinds of finite size effect (FSE): the standard FSE and that from fixed $Q$. The former effect is evaluated by the formulae determined by Colangelo et al. [49], which is developed from the Lüscher's formula for the relation between the pion scattering amplitude and the mass-shift in a finite box [50]. We use the NNLO results of Ref. [49] and the values of low energy constants estimated in Ref. [51]. For our lightest quark mass, they amount to $4.5 \%$ and $6.0 \%$ for $m_{\pi}^{2} / m_{q}$ and $f_{\pi}$, respectively. The FSE from the fixed topological charge is subject to Eq. (2.4). The $O\left(V^{-1}\right)$ corrections to $m_{\pi}$ and $f_{\pi}$ are determined at NLO of ChPT. The correction to $m_{\pi}$ starts at the tree-level. In the small quark mass region, it shifts the value of $m_{\pi}^{2} / m_{q}$ with the same size as the standard FSE while in the opposite direction. Thus the two effects almost cancel each other. The fixed- $Q$ correction to $f_{\pi}$ starts at NLO of ChPT, and gives small effect on the result of $f_{\pi}$.

Figure 5 shows the results of $m_{\pi}^{2} / m_{q}$ and $f_{\pi}$ after correcting FSEs. To compare with the ChPT expansion, we chose $\xi \equiv\left(m_{\pi} / 4 \pi f_{\pi}\right)^{2}$ as an expansion parameter, where $f_{\pi}$ is the measured value at each quark mass. We apply three types of ChPT fit: NLO, NNLO, and a simplified NNLO called NNLO' hereafter. The NNLO' formulae are obtained from those of NNLO by using an approximation $\xi^{2} \ln \xi \approx-2.5 \xi^{2}$, which is numerically reasonable in our target range, $0<\xi \lesssim 0.1$. Explicit functional forms are given in Ref. [39]. The results of fits are displayed in Figure 5. We 

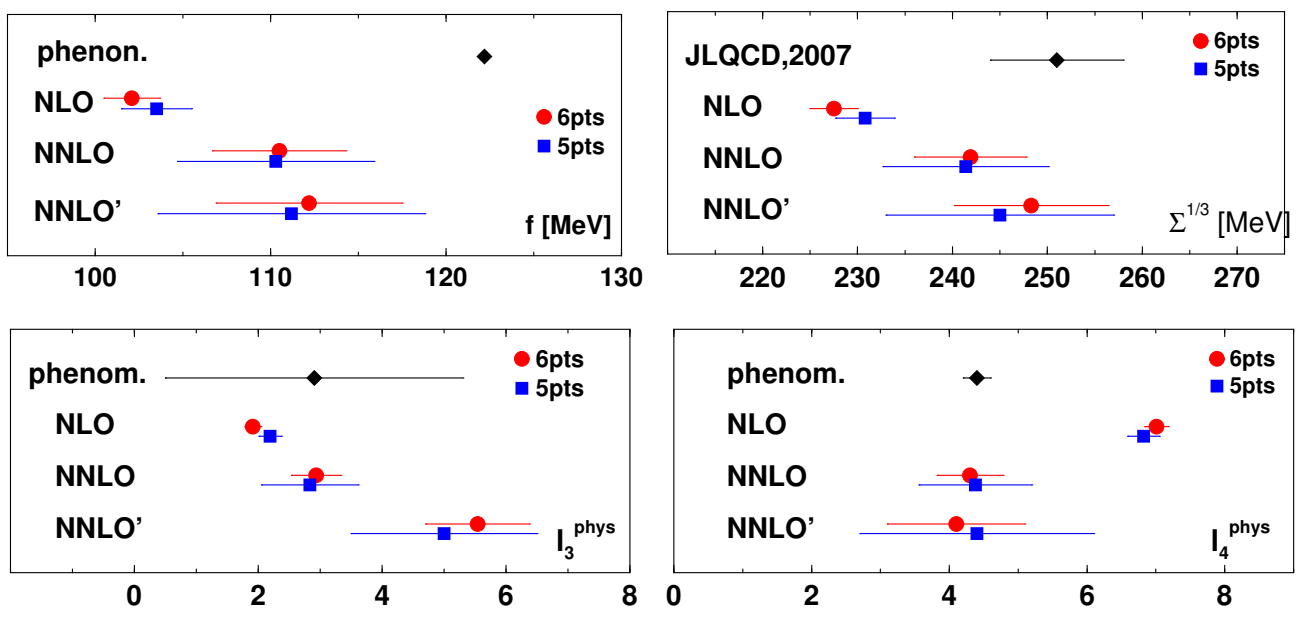

Figure 6: Comparisons of physical quantities obtained from the chiral fit ansatze: $f$ (upper left), $\Sigma^{1 / 3}$ (upper right), $l_{3}^{\text {phys }}$ (lower left) and $l_{4}^{\text {phys }}$ (lower right). In each panel, the red circles and blue squares are corresponding to the fits with 6 and 5 lightest data points, respectively.

note that the fit to the NNLO formulae is performed by a simultaneous fit for $m_{\pi}^{2} / m_{q}$ and $f_{\pi}$, and thus the value of $\chi^{2}$ cannot be compared directly to other fits.

These fits determine the following quantities: $f, \Sigma=B_{0} \cdot f^{2} / 2, l_{3}^{\text {phys }}$, and $l_{4}^{\text {phys }}$. Figure 6 compares the results of fits with the phenomenologically determined values ( $f[52]$ and $l_{3}^{\text {phys }} l_{4}^{\text {phys }}$ [51]). The result of $\Sigma$ is compared with our calculation in the $\varepsilon$-regime in Sec. 5.1 [34]. Except for the case of $l_{3}^{\text {phys }}$, the results of NLO fit are inconsistent with NNLO and the phenomenological estimates. This implies the failure of the NLO formulae to describe our data. The results of NNLO and NNLO' fits are consistent with each other and with the phenomenological estimates.

To quantify the FSEs more accurately, we need simulations with a larger lattice size, as well as a comparison with the result at $Q \neq 0$. We emphasize that these results indicate that the overlap simulations with fixed topology provide a framework for precision calculations of the spectrum and the matrix elements in the chiral regime.

\section{Conclusion}

We are performing large-scale simulations with $N_{f}=2$ and $N_{f}=2+1$ dynamical overlap fermions. The range of sea quark mass covers $m_{s}^{\text {phys }} / 6-m_{s}^{\text {phys }}$. The simulations are performed in fixed topological charge sectors. The results of the topological susceptibility and the pion mass and decay constant indicate that these simulations can provide a ground for precision computations of matrix elements with controlled chiral extrapolation.

We completed a generation of $N_{f}=2$ gauge configurations on $16^{3} \times 32$ lattices with $a \simeq 0.12$ $\mathrm{fm}$. Numbers of measurements are in progress and planned. The $N_{f}=2+1$ simulations on $16^{3} \times 48$ lattices are also in progress. These configurations will be supplied to ILDG soon after the first publication of the result of the spectrum.

For further investigation of finite size effects and for more extended objects than mesons, simulations at larger lattice sizes are desired. The target size of the spatial extent is 24 , which requires further improvements of numerical algorithms. 


\section{Acknowledgment}

Numerical simulations were performed on IBM System Blue Gene Solution and Hitachi SR11000 at High Energy Accelerator Research Organization (KEK) under a support of its Large Scale Simulation Program (No. 06-13, 07-16). We thank J. Doi, H. Samukawa, and S. Shimizu of IBM Japan Tokyo Research Laboratory for tuning the QCD code on the Blue Gene. The numerical simulations were partly performed on NEC SX8 at Yukawa Institute for Theoretical Physics, Kyoto University, and on NEC SX8 at Research Center for Nuclear Physics, Osaka University. The simulation also owes to a gigabit network SINET3 supported by National Institute of Informatics, for efficient data transfer supported by JLDG. This work is supported in part by the Grant-in-Aid of the Japanese Ministry of Education (No. 19740160).

\section{References}

[1] H. Neuberger, Phys. Lett. B 417 (1998) 141 [arXiv:hep-lat/9707022].

[2] H. Neuberger, Phys. Lett. B 427 (1998) 353 [arXiv:hep-lat/9801031].

[3] P. H. Ginsparg and K. G. Wilson, Phys. Rev. D 25 (1982) 2649.

[4] P. Hasenfratz, V. Laliena and F. Niedermayer, Phys. Lett. B 427 (1998) 125 [arXiv:hep-lat/9801021].

[5] M. Luscher, Phys. Lett. B 428 (1998) 342 [arXiv:hep-lat/9802011].

[6] P. M. Vranas, arXiv:hep-lat/0001006.

[7] H. Fukaya, arXiv:hep-lat/0603008.

[8] H. Fukaya, S. Hashimoto, K. I. Ishikawa, T. Kaneko, H. Matsufuru, T. Onogi and N. Yamada [JLQCD Collaboration], Phys. Rev. D 74 (2006) 094505 [arXiv:hep-lat/0607020].

[9] Z. Fodor, S. D. Katz and K. K. Szabo, JHEP 0408 (2004) 003 [arXiv:hep-lat/0311010].

[10] T. Kaneko et al. [JLQCD Collaboration], PoS LAT2006 (2006) 054 [arXiv:hep-lat/0610036].

[11] S. Hashimoto et al. [JLQCD Collaboration], PoS LAT2006 (2006) 052 [arXiv:hep-lat/0610011].

[12] M. Golterman and Y. Shamir, arXiv:0705.2928 [hep-lat].

[13] R. Brower, S. Chandrasekharan, J. W. Negele and U. J. Wiese, Phys. Lett. B 560 (2003) 64 [arXiv:hep-lat/0302005].

[14] S. Aoki, H. Fukaya, S. Hashimoto and T. Onogi, Phys. Rev. D 76 (2007) 054508 [arXiv:0707.0396 [hep-lat]].

[15] T. Onogi et al., PoS (LATTICE 2007) 080.

[16] J. van den Eshof, A. Frommer, T. Lippert, K. Schilling and H. A. van der Vorst, Comput. Phys. Commun. 146 (2002) 203 [arXiv:hep-lat/0202025].

[17] T. W. Chiu, T. H. Hsieh, C. H. Huang and T. R. Huang, Dirac operator," Phys. Rev. D 66 (2002) 114502 [arXiv:hep-lat/0206007].

[18] A. Frommer, B. Nockel, S. Gusken, T. Lippert and K. Schilling, Int. J. Mod. Phys. C 6 (1995) 627 [arXiv:hep-lat/9504020].

[19] B. Jegerlehner, arXiv:hep-lat/9612014. 
[20] N. Cundy, J. van den Eshof, A. Frommer, S. Krieg, T. Lippert and K. Schafer, Comput. Phys. Commun. 165 (2005) 221 [arXiv:hep-lat/0405003].

[21] A. Borici, arXiv:hep-lat/0402035.

[22] R. G. Edwards, B. Joo, A. D. Kennedy, K. Orginos and U. Wenger, PoS LAT2005 (2006) 146 [arXiv:hep-lat/0510086].

[23] S. Hashimoto et al. [JLQCD Collaboration], PoS (LATTICE 2007) 101.

[24] Y. Aoki et al., Phys. Rev. D 72 (2005) 114505 [arXiv:hep-lat/0411006].

[25] M. Hasenbusch, Phys. Lett. B 519 (2001) 177 [arXiv:hep-lat/0107019].

[26] J. C. Sexton and D. H. Weingarten, Nucl. Phys. B 380 (1992) 665.

[27] A. D. Kennedy and J. Kuti, Phys. Rev. Lett. 54 (1985) 2473.

[28] H. Matsufuru et al. [JLQCD Collaboration], PoS LAT2006 (2006) 031 [arXiv:hep-lat/0610026].

[29] A. Bode, U. M. Heller, R. G. Edwards and R. Narayanan, arXiv:hep-lat/9912043.

[30] T. DeGrand and S. Schaefer, JHEP 0607 (2006) 020 [arXiv:hep-lat/0604015].

[31] J. Doi, PoS (LATTICE 2007) 032.

[32] N. Yamada et al. [JLQCD Collaboration], PoS LAT2006 (2006) 060 [arXiv:hep-lat/0609073].

[33] H. Fukaya et al. [JLQCD Collaboration], PoS LAT2006 (2006) 050 [arXiv:hep-lat/0610024].

[34] H. Fukaya et al. [JLQCD Collaboration], Phys. Rev. Lett. 98 (2007) 172001 [arXiv:hep-lat/0702003].

[35] H. Fukaya et al., Phys. Rev. D 76 (2007) 054503 [arXiv:0705.3322 [hep-lat]].

[36] H. Fukaya et al. [JLQCD Collaboration], PoS (LATTICE 2007) 073.

[37] T-W. Chiu et al. [TWQCD and JLQCD Collaboration], PoS (LATTICE 2007) 068.

[38] S. Aoki et al. [JLQCD-TWQCD Collaboration], arXiv:0710.1130 [hep-lat].

[39] J. Noaki et al. [JLQCD Collaboration], PoS (LATTICE 2007) 126.

[40] T. Kaneko et al. [JLQCD Collaboration], PoS (LATTICE 2007) 148.

[41] N. Yamada et al. [JLQCD Collaboration], PoS (LATTICE 2007) 379.

[42] E. Shintani et al. [JLQCD Collaboration], PoS (LATTICE 2007) 134.

[43] T. Yagi et al., PoS (LATTICE 2007) 086.

[44] T. Banks and A. Casher, Nucl. Phys. B 169 (1980) 103.

[45] T. A. DeGrand and S. Schaefer, Comput. Phys. Commun. 159 (2004) 185 [arXiv:hep-lat/0401011].

[46] L. Giusti, P. Hernandez, M. Laine, P. Weisz and H. Wittig, JHEP 0404 (2004) 013 [arXiv:hep-lat/0402002].

[47] H. Leutwyler and A. Smilga, Phys. Rev. D 46 (1992) 5607.

[48] G. Martinelli, C. Pittori, C. T. Sachrajda, M. Testa and A. Vladikas, Nucl. Phys. B 445 (1995) 81 [arXiv:hep-lat/9411010].

[49] G. Colangelo, S. Durr and C. Haefeli, Nucl. Phys. B 721 (2005) 136 [arXiv:hep-lat/0503014].

[50] M. Luscher, Commun. Math. Phys. 104 (1986) 177.

[51] G. Colangelo, J. Gasser and H. Leutwyler, Nucl. Phys. B 603 (2001) 125 [arXiv:hep-ph/0103088].

[52] J. Gasser and H. Leutwyler, Annals Phys. 158 (1984) 142. 